\title{
Redescription of Prosthenhystera obesa (Diesing, 1850) (Callodistomidae, Digenea) with New Host Records and Data on Morphological Variability
}

\author{
Anna Kohn $/ * /+$, Berenice MM Fernandes/*, \\ Maria de Fatima D Baptista-Farias
}

\begin{abstract}
Laboratório de Helmintos Parasitos de Peixes, Departamento de Helmintologia, Instituto Oswaldo Cruz, Av. Brasil 4365, 21045-900 Rio de Janeiro, RJ, Brasil
\end{abstract}

Prosthenhystera obesa (Diesing,1850) Travassos, 1922 from the gall bladder of Astyanax bimaculatus, Caranx gibbosus, Galeocharax humeralis, Leporinus copelandii, Pimelodus fur, Pseudopimelodus roosevelti, Salminus brevidens, Salminus maxillosus and from the new hosts, Cynopotamus amazonum and Triurobrycon lundii is redescribed, demonstrating a large morphological variation, mainly in body and testes size and shape. New hosts harbouring immature specimens of P. obesa are presented: Brycon $s p$., Leporellus vittatus, Pachyurus squamipinnis, Pimelodus clarias, Pseudoplatystoma corruscans and Salminus hilarii. Scanning electron microscopy micrographies, original figures and measurements of adult and immature specimens from different Brazilian hosts and localities are presented.

Key words: Prosthenhystera obesa - scanning electron microscopy - freshwater fish parasites - Brazil

Prosthenhystera obesa (Diesing, 1850) was well described by Travassos (1922b) and Travassos et al. (1928) and also recorded by other authors from different hosts and localities in Brazil (Travassos \& Freitas 1941, Travassos \& Kohn 1965, Kohn \& Fernandes 1981, 1987, Pavanelli et al. 1992), Mexico (Caballero \& Jimmenez 1969) and Colombia (Thatcher 1991).

The study of a large number of specimens, collected mainly by Travassos since 1918, from different hosts and localities in Brazil and from specimens of Salminus maxillosus collected by the authors from the Paraná River, in the localities of Guaira in 1985 and Foz do Iguaçu in 1991, allowed the redescription of the species and the demonstration of the large morphological variability of this parasite.

\section{MATERIALS AND METHODS}

Fish were collected with nets and kept alive until they were examined. Prior to light microscopy, the specimens were fixed in AFA (alcohol, formalin, acetic acid) under slight coverslip pressure, stained in alcoholic-acid carmine, dehydrated

\footnotetext{
* Research fellow from Conselho Nacional de Desenvolvimento Científico e Tecnológico - CNPq ${ }^{+}$Corresponding author. Fax: +55-21-260.4866/590.3545 E-mail: annakohn@marlin.com.br Received 5 February 1996 Accepted 27 November 1996
}

in an alcohol series and mounted in Canada balsam. Measurements were made using a calibrated filar micrometer and are given in micrometres. For scanning electron microscopy (SEM), specimens were previously fixed in glutaraldeyde $2.5 \%$, postfixed for $1 \mathrm{hr}$ with $1 \%$ osmium tetroxide in $0.1 \mathrm{M}$ phosphate buffer, dehydrated in graded ethanol, critical point dried using $\mathrm{CO}_{2}$, and coated with gold. The observations were made using a Zeiss DSM 940 scanning electron microscope. Eighty six specimens from different hosts and localities, were studied and deposited in the Helminthological Collection of the Oswaldo Cruz Institute (CHIOC). Part of the material deposited by Travassos in the CHIOC, was preserved in Railliet and Henry's fluid, and part on whole mounts.

\section{RESULTS}

\section{Prosthenhystera obesa (Diesing,1850) \\ Travassos, 1922}

Figs 1-7

Synonym: Pseudoprosthenhystera microtesticulata Kloss, 1966

Hosts: Astyanax bimaculatus (Linnaeus) (= Cichlasoma bimaculatum (L); Brycon $\mathrm{sp}$. (new host record); Caranx gibbosus (Linnaeus); Cynopotamus amazonum (Guenther) (new host record); Galeocharax humeralis (Valenciennes) (= Cynopotamus humeralis, Acestrorhamphus sp.); Leporellus vittatus Valenciennes (new host record); Leporinus copelandii Steindachner; Pachyurus squamipinnis Agassiz (new host record); Pimelodus clarias Linnaeus (new host record); 

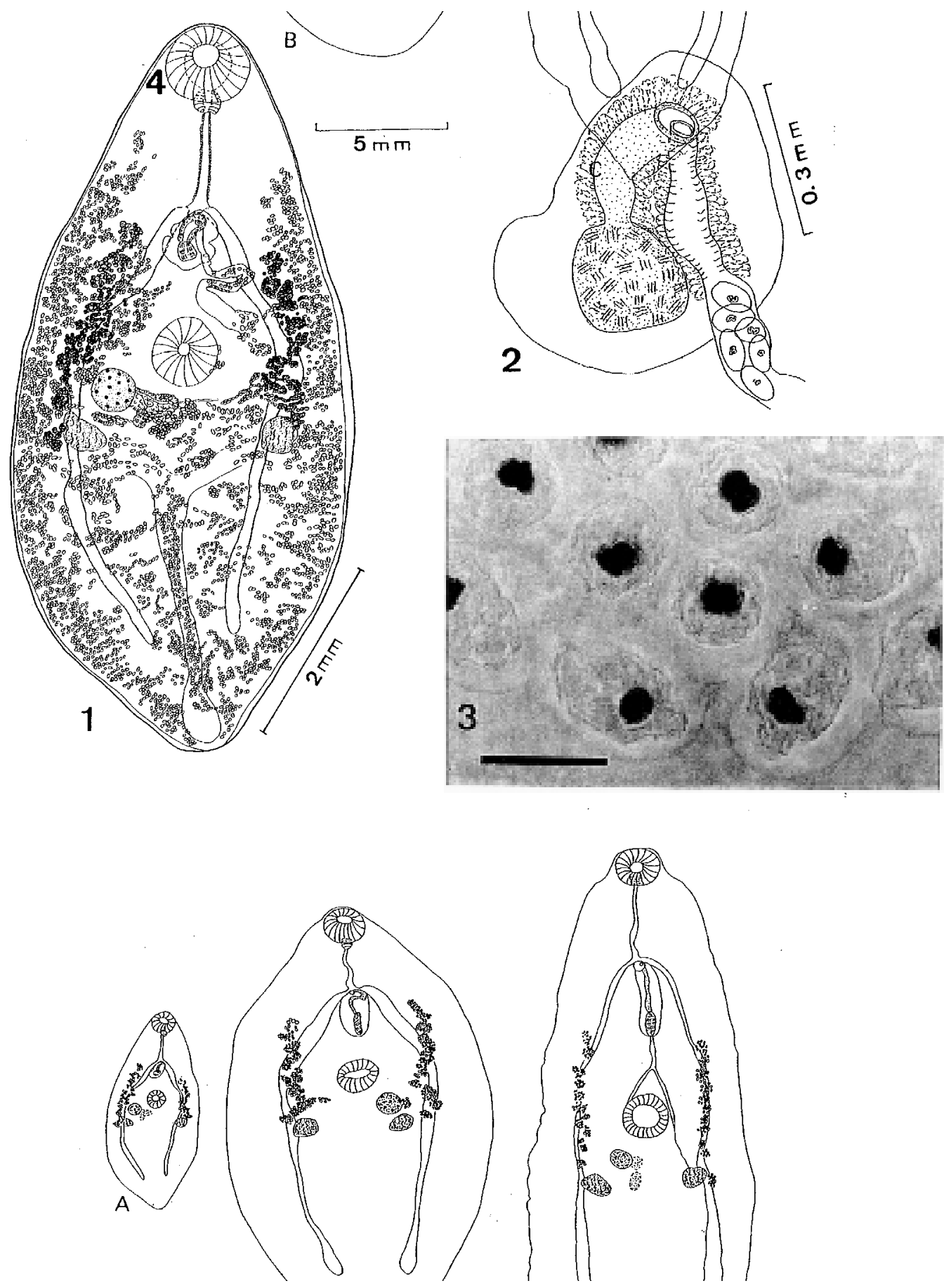

Prosthenhystera obesa from Salminus maxillosus. Fig. 1: total view, no. 33.243b. Fig. 2: terminal genitalia no. 33.243b. Fig. 3: photomicrography of eggs, no. 33.244 , bar $=0.04 \mathrm{~mm}$. Figs 4 A-C (figs in same scale): total view of mature specimens (uterus not represented) showing the large variation of body size: 4A: no. 33.243b, 4B: no. 33.244, 4C: no. 33.246 . 

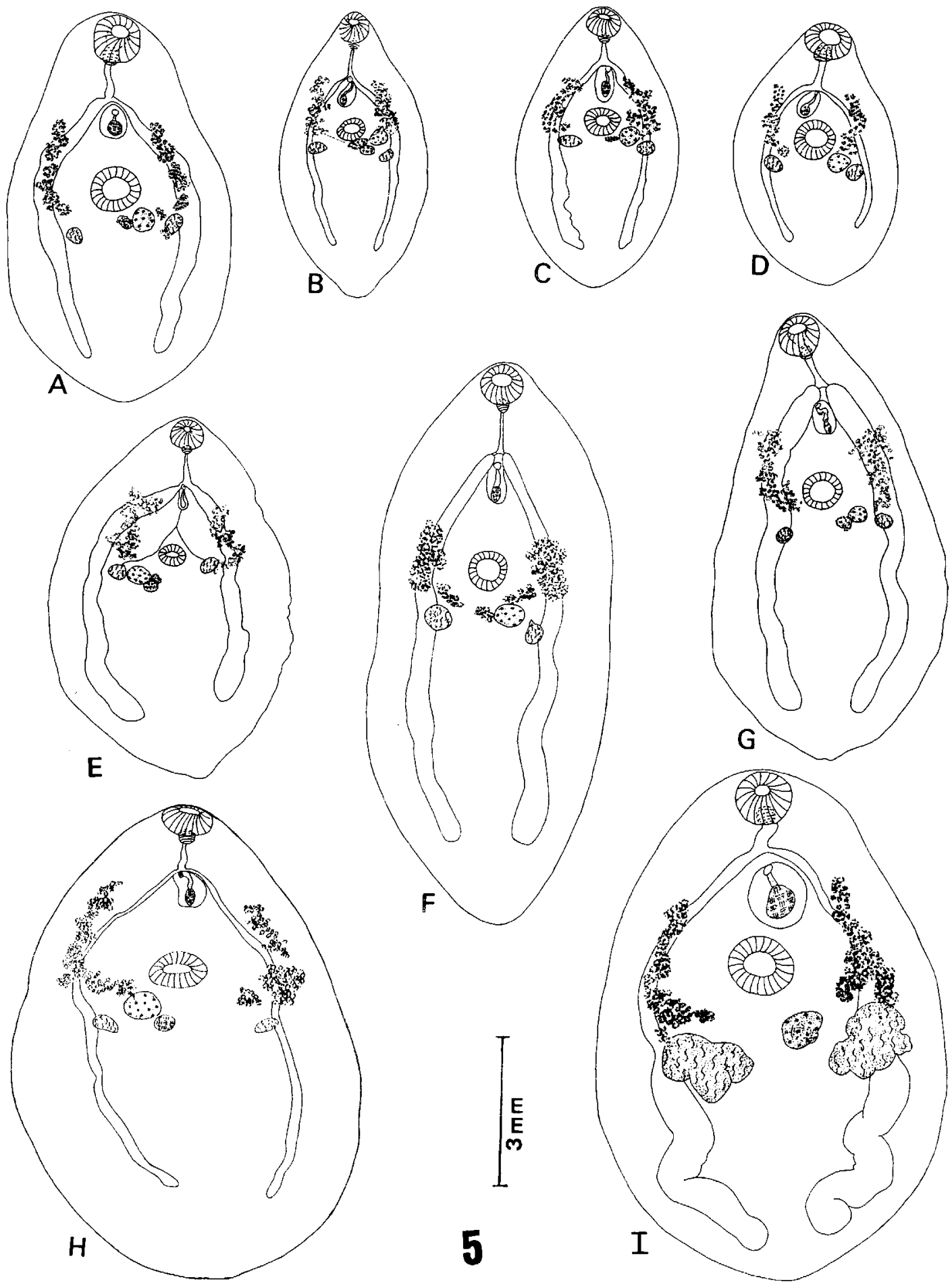

Fig. 5 (figs in same scale): Prosthenhystera obesa. Total view of mature specimens (uterus not represented) demonstrating variation in body and testes size and shape of specimens from different hosts. A: no. 16.563 from Triurobrycon lundii. B: no. 31.818a from Caranx gibbosus. C: no. 33.234 from Cynopotamus amazonum. D: no. 33.256 from Pseudopimelodus roosevelti. E: no. 11.270 from Astyanax bimaculatus. F: no. 33.266 from Galeocharax humeralis. G: no. 33.240 from Leporinus copelandii. H: no. 33.252a from Salminus brevidens. I: no. 16.231 from Pseudopimelodus roosevelti. 
Pimelodus fur (Lutk); Pseudopimelodus roosevelti Borodin; Pseudoplatystoma corruscans (Agassiz) (new host record); Salminus brevidens (Cuvier); Salminus hilarii Cuvier \& Valenciennes (new host record); Salminus maxillosus (Cuvier \& Valenciennes); Triurobrycon lundii Reinhardt (new host record).

Site in hosts: gall bladder.

Data on the morphometric variation of 49 adult specimens are summarized in Tables I and II and of 21 immature specimens are presented in Table III.

Redescription: body flattened, usually oval, may be elliptical to round with rounded posterior end, narrowing in forebody, with large variation in size. Oral sucker rounded, subterminal. Ventral sucker muscular, pre-equatorial. Suckers nearly of the same size, width ratio within the range of 1:0.8-1:1. Pharynx small, rounded. Oesophagus long, slender, surrounded by glandular cells, extending back to about the level of genital pore; at this point the gut bifurcates, originating two narrow caeca, slightly sinuous, ending blindly near the posterior extremity of the body. Testes two, irregular in shape, usually smooth and smaller than ovary, may also be lobed and larger than ovary, lateral, symmetrically situated in ovarian zone or below, intracaecal, caecal or extracaecal. Vas efferentia extending from anteromedial margin of testes to form short vas deferens in front of acetabular level; vas deferens entering seminal vesicle at the posterior region of cirrus-sac. Cirrus-sac median, between two suckers, extending back from genital pore, may reach pre-acetabular zone, contains saccular or elongated seminal vesicle, pars prostatica tubular surrounded by gland cells and well developed ejaculatory duct. Genital atrium small; common genital pore mid-ventral, immediately below oesophageal bifurcation. Ovary rounded to oval, posterolateral to acetabulum, equatorial. A large Mehlis' gland and a well developed seminal receptacle lie next to ovary; the oviduct receives the common vitelline duct and the Laurer's canal which opens dorsally. Vitellaria follicular, in two lateral fields, caecal, intracaecal and extracaecal, may extend from ovary level to mid-forebody. Uterus coiled, intracaecal, caecal and extracaecal, filling most of hindbody in mature specimens, reaching the posterior extremity, extending to forebody, to level of oral sucker, opening into genital atrium through the muscular metraterm, which is surrounded by glands. Eggs very small, oval, oper- culated, present miracidium with an irregular "v" or "8" shaped black spot (Fig. 3). Excretory vesicle long and wide, Y-shaped. The excretory pore opens at the posterior end of the body.

One specimen from S. maxillosus from the Paraná River observed by SEM, showed round shaped body (Fig. 6) and tegument with aciliated papillae (Fig. 7) irregularly distributed on the surface, more evident around the oral sucker and not observed around the acetabulum, genital and excretory pores.
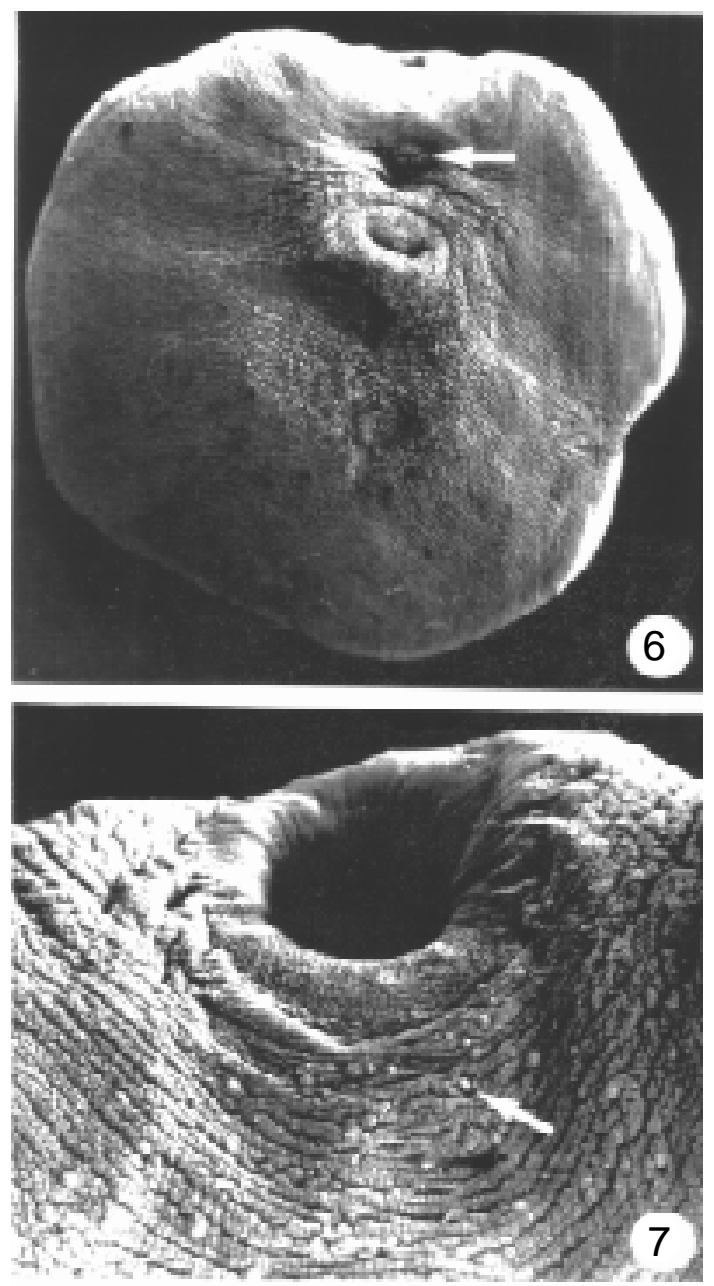

Scanning electron micrographs of Prosthenhystera obesa. Fig. 6: whole mount (ventral view), X 22. Fig. 7: anterior end of body with oral sucker showing ridge tegument and buttonlike papillae (arrow), X 150. 
TABLE I

Original measurements (in mm) of Prosthenhystera obesa from Salminus maxillosus from different localities

\begin{tabular}{|c|c|c|c|c|c|c|c|c|}
\hline CHIOC no. & 2.147 & $3.279,33.249$ & $33.246,33.253$ & $33.247,33.248$ & $16.220,16.224$ & $33.252,33.254$ & 33.243 & 33.244 \\
\hline Specimens measured & 1 & 2 & 2 & 2 & 2 & 3 & 2 & 1 \\
\hline Body L & 14,027 & $11,092-15,040$ & $16,315-23,160$ & $8,757-17,412$ & $13,615-17,712$ & $9,517-17,900(12,349)$ & $7,740-7,860$ & 15,977 \\
\hline Body W & 8,157 & $4,960-7,632$ & $6,545-8,570$ & $6,507-7,632$ & $9,367-10,530$ & $6,170-9,930(7,561)$ & $3,497-3,572$ & 10,155 \\
\hline Oral sucker L & 1,305 & $1,045-1,091$ & $1,387-1,530$ & $1,076-1,137$ & $1,137-1,290$ & $566-1,499(970)$ & $733-769$ & 1,259 \\
\hline Oral sucker W & 1,244 & $1,045-1,183$ & $1,484-1,660$ & $1,091-1,395$ & $1,275-1,305$ & $877-1,407(1,079)$ & $806-879$ & 1,499 \\
\hline Ventral sucker L & 1,290 & $908-1,290$ & $1,500-1,537$ & $1,015-1,302$ & $1,091-1,244$ & $709-1,484(1,023)$ & $659-711$ & 1,183 \\
\hline Ventral sucker W & 1,259 & $1,030-1,350$ & $1,350-1,660$ & $1,122-1,422$ & $1,198-1,275$ & $908-1,575(1,181)$ & $689-696$ & 1,575 \\
\hline Sucker width ratio & $1: 1$ & $1: 1$ & $1: 0.9-1: 1$ & $1: 1$ & $1: 0.9-1: 1$ & $1: 1$ & $1: 0.8$ & $1: 1$ \\
\hline Pharynx L & 361 & $260-407$ & $271-453$ & 466 & $336-438$ & $229-535(336)$ & $251-306$ & 392 \\
\hline Pharynx W & 321 & $392-469$ & $271-484$ & 354 & $453-469$ & $260-469(335)$ & $242-275$ & 242 \\
\hline Oesophagus L & 1,290 & $724-647$ & $1,591-2,785$ & $535-1,244$ & $678-1,499$ & $392-1,214(692)$ & $738-939$ & 1,468 \\
\hline Seminal receptacle $\mathrm{L}$ & 336 & 275 & - & 275 & $290-306$ & $321-825(538)$ & 184 & - \\
\hline Seminal receptacle W & 484 & 275 & - & 392 & $469-535$ & $321-525(423)$ & 122 & - \\
\hline Cirrus-sac L & 1,805 & 1,259 & 2,822 & $1,321-1,545$ & 1,637 & $632-739$ & 602 & 1,606 \\
\hline Cirrus-sac W & 1,076 & 571 & 750 & $938-1,244$ & 1,336 & $550-678$ & 587 & 969 \\
\hline Seminal vesicle L & 617 & 602 & - & - & - & $260-392$ & $199-251$ & 785 \\
\hline Seminal vesicle $\mathrm{W}$ & 137 & 306 & - & - & - & $183-229$ & $139-199$ & 306 \\
\hline Testes L & $586-602$ & $535-602$ & $709-900$ & $846-1,061$ & $423-816$ & $244-787(460)$ & $326-480$ & $602-617$ \\
\hline Testes W & $602-663$ & $514-755$ & $750-1,000$ & $602-1,407$ & $724-1,106$ & $469-1,050(695)$ & $298-420$ & $862-877$ \\
\hline Ovary L & 908 & 678 & $525-663$ & $520-816$ & $785-1,015$ & $602-787(664)$ & $457-480$ & 693 \\
\hline Ovary W & 816 & 816 & $562-755$ & $647-785$ & $908-1,061$ & $709-900(788)$ & $480-494$ & 938 \\
\hline Eggs L & $73-76$ & $49-75(58)$ & $60-64(62)$ & $60-75(68)$ & $56-66(62)$ & $60-75(65)$ & $58-81(70)$ & $52-64(57)$ \\
\hline Eggs W & $47-48$ & $30-45(34)$ & $37-41(39)$ & $34-41(37)$ & $30-35(34)$ & $34-45(38)$ & $36-40(38)$ & $37-41(38)$ \\
\hline Locality & $\begin{array}{c}\text { Tibiriçáa } \\
\text { São Paulo }\end{array}$ & $\begin{array}{c}\text { Lassance } \\
\text { Minas Gerais }\end{array}$ & $\begin{array}{l}\text { Porto Esperança } \\
\text { Mato Grosso }\end{array}$ & $\begin{array}{l}\text { Pirassununga } \\
\text { São Paulo }\end{array}$ & $\begin{array}{l}\text { Pirassununga } \\
\text { São Paulo }\end{array}$ & $\begin{array}{c}\text { Pirapora } \\
\text { Minas Gerais }\end{array}$ & $\begin{array}{l}\text { Guaira } \\
\text { Paraná }\end{array}$ & $\begin{array}{c}\text { Foz do Iguaçu } \\
\text { Paraná }\end{array}$ \\
\hline Date of collection & 1918 & 1921 & 1922 & 1927 & 1946 & 1958 & 1985 & 1991 \\
\hline
\end{tabular}

CHIOC: Helminthological Collection of the Oswaldo Cruz Institute; L: length; W: width 
TABLE II

Original measurements (in $\mathrm{mm}$ ) of Prosthenhystera obesa from different hosts and localities

\begin{tabular}{|c|c|c|c|c|c|c|c|c|}
\hline CHIOC no. & $11.270,11.271,11.272$ & 31.818 & 33.234 & $33.263,33.264,33.266$ & $16.567,33.240,33.241$ & $\begin{array}{l}16.230-16.236,16.238 \\
33.255,33.256-33.258\end{array}$ & $33.242,33.245$ & 16.563 \\
\hline Specimens measured & 3 & 2 & 1 & 6 & 4 & 13 & 4 & 1 \\
\hline Body L & $7,196-7,242$ & $5,340-5,420$ & 5,207 & $6,770-10,792(7,934)$ & $5,795-9,367(6,716)$ & 4,960-14,665 (10,099) & $7,782-12,330(10,112)$ & 7,332 \\
\hline Body W & $4,575-4,952(4,721)$ & $2,820-3,160$ & 3,075 & $2,485-4,397(3,422)$ & $4,285-5,110(4,866)$ & $3,085-8,007(5,737)$ & $5,823-9,517(7,463)$ & 3,988 \\
\hline Oral sucker L & $566-587$ & $510-570$ & 550 & 484-755 (610) & $550-785(676)$ & $678-1,515(1,149)$ & $1,015-1,393(1,215)$ & 908 \\
\hline Oral sucker W & $602-678$ & $560-570$ & 644 & $550-816(667)$ & $571-831(728)$ & $938-1,545(1,271)$ & $1,122-1,484(1,284)$ & 908 \\
\hline Ventral sucker L & $484-515(489)$ & $382-440$ & 531 & $475-724(580)$ & $566-693(626)$ & $737-1,407(1,116)$ & $1,015-1,321(1,202)$ & 816 \\
\hline Ventral sucker W & $515-520(517)$ & $460-499$ & 709 & $438-724(600)$ & $586-709(628)$ & $766-1,422(1,168)$ & $1,107-1,545(1,345)$ & 923 \\
\hline Sucker width ratio & $1: 0.8$ & $1: 0.8-0.9$ & $1: 1.1$ & $1: 0.8-1.0$ & $1: 0.8-1: 1$ & $1: 0.8-1: 1$ & $1: 1$ & $1: 1$ \\
\hline Pharynx L & $158-168(164)$ & $170-220$ & 158 & $186-275(227)$ & $168-223(186)$ & 244-484 (383) & 377 & 229 \\
\hline Pharynx W & 183-198 (189) & $170-220$ & 214 & $198-290(252)$ & $168-225$ (197) & $290-550(429)$ & 438 & 306 \\
\hline Oesophagus L & $469-587$ & $354-535$ & 345 & $306-831(510)$ & $183-709$ (498) & $469-1,137(795)$ & $469-535$ & 678 \\
\hline Seminal receptacle L & $137-183$ & $170-180$ & - & $177-535$ & 392 & $229-632(379)$ & 183-484 (379) & - \\
\hline Seminal receptacle $\mathrm{W}$ & $260-306$ & $210-240$ & - & $198-233$ & 571 & $362-862(562)$ & $275-306(296)$ & - \\
\hline Cirrus-sac L & $466-468$ & $429-541$ & 634 & $541-785(658)$ & $205-447$ & $606-1,259(922)$ & $801-1,392(1,154)$ & 586 \\
\hline Cirrus-sac W & $111-373$ & $205-223$ & 298 & $205-469(362)$ & $166-279$ & $289-862(561)$ & $770-999(885)$ & 566 \\
\hline Seminal vesicle $\mathrm{L}$ & 120 & $142-176$ & 335 & $186-382(264)$ & 225 & $186-770(487)$ & $408-647$ & 233 \\
\hline Seminal vesicle $\mathrm{W}$ & 75 & $75-127$ & 242 & $121-321(170)$ & 48 & $168-984(773)$ & $153-275$ & 214 \\
\hline Testes L & $290-321$ (309) & $190-200$ & $242-261$ & $214-770(520)$ & $289-514(342)$ & $260-877(624)$ & $306-659$ (419) & $275-306$ \\
\hline Testes W & $306-361$ (327) & $290-350$ & $354-401$ & $261-800(674)$ & $233-571(418)$ & $321-1,168(807)$ & $423-816(547)$ & $306-336$ \\
\hline Ovary L & $306-392(345)$ & $290-320$ & 373 & $307-484(385)$ & $321-438(361)$ & $336-785$ (613) & $469-484$ (476) & 453 \\
\hline Ovary W & $392-514(448)$ & $350-420$ & 373 & $391-602$ (489) & $354-484(421)$ & $407-1,030(675)$ & $648-709(682)$ & 438 \\
\hline Eggs L & $51-75(62)$ & $56-67(63)$ & $59-68(63)$ & $47-62(56)$ & $58-87(69)$ & $52-73(60)$ & $67-75(71)$ & $67-79(73)$ \\
\hline Eggs W & $23-37(27)$ & $34-37(35)$ & $23-30(27)$ & $35-48(40)$ & $35-59(44)$ & $31-52(37)$ & $37-45(41)$ & $36-40(37)$ \\
\hline Host & $\begin{array}{c}\text { Astyanax } \\
\text { bimaculatus }\end{array}$ & $\begin{array}{l}\text { Caranx } \\
\text { gibbosus }\end{array}$ & $\begin{array}{c}\text { Cynopotamus }^{a} \\
\text { amazonum }^{a}\end{array}$ & $\begin{array}{c}\text { Galeocharax } \\
\text { humeralis }\end{array}$ & $\begin{array}{l}\text { Leporinus } \\
\text { copelandii }\end{array}$ & $\begin{array}{c}\text { Pseudopimelodus } \\
\text { roosevelti }\end{array}$ & $\begin{array}{l}\text { Salminus } \\
\text { brevidens }\end{array}$ & $\begin{array}{c}\text { Triurobrycon }^{a} \\
\text { lundii }\end{array}$ \\
\hline Locality & $\begin{array}{l}\text { Ilha Seca } \\
\text { São Paulo }\end{array}$ & $\begin{array}{c}\text { Salobra } \\
\text { Mato Grosso }\end{array}$ & $\begin{array}{c}\text { Foz do Iguaçu } \\
\text { Paraná }\end{array}$ & $\begin{array}{l}\text { Pirassununga } \\
\text { São Paulo }\end{array}$ & $\begin{array}{l}\text { Pirassununga } \\
\text { São Paulo }\end{array}$ & $\begin{array}{l}\text { Pirassununga } \\
\text { São Paulo }\end{array}$ & $\begin{array}{c}\text { Barra, Rio Grande } \\
\text { Bahia }\end{array}$ & $\begin{array}{r}\text { Pirassununga } \\
\text { São Paulo }\end{array}$ \\
\hline Date of collection & 1940 & 1941 & 1994 & 1962 & 1946 & 1962 & 1962 & 1946 \\
\hline
\end{tabular}

CHIOC: Helminthological Collection of the Oswaldo Cruz Intitute; $a$ : new host record; L: length; W: width 
TABLE III

Original measurements (in $\mathrm{mm}$ ) of immature specimens of Prosthenhystera obesa from different hosts and localities

\begin{tabular}{|c|c|c|c|c|c|c|c|c|}
\hline CHIOC no. & $16.988,16.997$ & 33.260 & $\begin{array}{c}16.989,16.990, \\
16.992,16.998\end{array}$ & $\begin{array}{l}5.596,12.028, \\
33.231-33.233\end{array}$ & 11.269 & 33.261 & $33.239,33.250 .33 .251$ & 33.262 \\
\hline Specimens measured & 4 & 1 & 4 & 4 & 1 & 1 & 5 & 1 \\
\hline Body L & $1,560-3,381(2,578)$ & 2,642 & $1,468-2,376(1,923)$ & $3,102-6,573(4,912)$ & 3,010 & 7,120 & $2,972-6,441(5,155)$ & 3,880 \\
\hline Body W & $1,015-2,285(1,436)$ & 1,501 & $923-2,030(1,374)$ & $1,852-3,228(2,586)$ & 2,500 & 3,180 & $1,612-4,247(2,985)$ & 1,390 \\
\hline Oral sucker L & $261-484(351)$ & 373 & $251-345(286)$ & $447-550$ & 469 & 831 & $499-831(731)$ & 466 \\
\hline Oral sucker W & $298-499(389)$ & 373 & $279-345(307)$ & $503-678$ & 514 & 831 & $499-862(731)$ & 466 \\
\hline Ventral sucker L & $242-336(283)$ & - & $242-279(258)$ & $522-632$ & 520 & 816 & $469-800(697)$ & 513 \\
\hline Ventral sucker W & $289-306(295)$ & - & $242-317(272)$ & $522-693$ & 535 & 755 & $469-800(697)$ & 513 \\
\hline Sucker width ratio & $1: 0.7-1$ & - & $1: 0.8-0.9$ & $1: 1$ & $1: 1$ & $1: 0.9$ & $1: 0.9-1$ & $1: 1$ \\
\hline Pharynx L & $102-153$ & 139 & $84-111(96)$ & $168-214(188)$ & 153 & 275 & $137-244(180)$ & - \\
\hline Pharynx W & $112-137$ & 121 & $102-135(110)$ & $168-205(185)$ & 168 & 290 & $153-260(205)$ & - \\
\hline Oesophagus L & $158-514$ & 298 & $84-382(202)$ & $298-1,137$ (654) & 392 & 678 & $307-571(470)$ & 447 \\
\hline Cirrus-sac L & - & - & 186 & $183-198$ & - & 739 & $102-550(416)$ & - \\
\hline Cirrus-sac W & - & - & 130 & $107-183$ & - & 306 & $111-423(298)$ & - \\
\hline Testes L & $56-229(135)$ & - & $102-233(155)$ & $60-97$ & - & $229-260$ & $56-407(235)$ & - \\
\hline Testes W & $75-244(143)$ & - & $93-195(144)$ & $75-78$ & - & $198-244$ & $45-453(307)$ & - \\
\hline Ovary L & $92-275$ & - & $102-214(142)$ & - & - & 260 & $48-275(159)$ & - \\
\hline Ovary W & $76-244$ & - & $111-214(149)$ & - & - & 198 & $86-321(182)$ & - \\
\hline Host & Brycon sp. ${ }^{a}$ & $\begin{array}{l}\text { Leporellus }^{a} \\
\text { vittatus }\end{array}$ & $\begin{array}{l}\text { Pachyurus }^{a} \\
\text { squamipinnis }\end{array}$ & $\begin{array}{l}\text { Pimelodus }^{a} \\
\quad \text { clarias }\end{array}$ & $\begin{array}{l}\text { Pimelodus } \\
\quad \text { fur }\end{array}$ & $\begin{array}{c}\text { Pseudoplatystoma } \\
\text { corruscans }\end{array}$ & $\begin{array}{l}\text { Salminus } \\
\text { brevidens }\end{array}$ & $\begin{array}{l}\text { Salminus }^{a} \\
\text { hilarii }\end{array}$ \\
\hline Locality & $\begin{array}{l}\text { Lagoa Juparanã } \\
\text { Espírito Santo }\end{array}$ & $\begin{array}{l}\text { Pirassununga } \\
\text { São Paulo }\end{array}$ & $\begin{array}{l}\text { Lagoa de Juparanã } \\
\text { Espírito Santo }\end{array}$ & $\begin{array}{l}\text { Porto Esperança } \\
\text { Mato Grosso }\end{array}$ & $\begin{array}{l}\text { Ilha Seca } \\
\text { São Paulo }\end{array}$ & $\begin{array}{c}\text { Pirapora } \\
\text { Minas Gerais }\end{array}$ & $\begin{array}{c}\text { Pirapora } \\
\text { Minas Gerais }\end{array}$ & $\begin{array}{r}\text { irassununga } \\
\text { São Paulo }\end{array}$ \\
\hline Date of collection & 1948 & 1947 & 1948 & 1925 & 1940 & 1957 & 1957 & 1962 \\
\hline
\end{tabular}

CHIOC: Helminthological Collection of the Oswaldo Cruz Institute; $a$ : new host record; L: length; W: width 


\section{DISCUSSION}

Diesing $(1850,1855)$ reported Distomum obesa from specimens collected by Natterer from Salminus brevidens and Leporinus friderici from the State of Mato Grosso and from Xiphostoma cuvieri found in the State of Acre, Brazil, with the following description.

"Corpus ellipticum erassum, supra planum, subtus ventricosum. Os subterminale anticum circulare. Acetabulum magnitudine oris, subcentrale superum, apertura circulari. Penis retractus, apertura genitali ampla, in medio inter os et acetabulum. Longit. 3-7"'; latit. 2-5"'; erassit. $11 / 2$ '"'”.

(Paiva in 1983 referred that the commonly named fish "dourado" from the São Francisco River belongs to the species Salminus brevidens and the ones from Southeast, Center-West and South of Brazil (Paraná and Paraguai River basins), belong to Salminus maxillosus. Considering this, S. brevidens mentioned by Diesing $(1850,1855)$ from the State of Mato Grosso and by Travassos (1922a, 1922b) from the States of Mato Grosso and São Paulo, belongs to S. maxillosus.)

In 1920, during a meeting of the Brazilian Society of Sciences, Travassos proposed the new genus Prosthenhystera for D. obesum, with a description, without figures, of specimens from Salminus maxillosus (= S. brevidens) and Leporinus sp. from "Tibiriçá", State of São Paulo (Travassos 1922a). In another paper, Travassos (1922b) presented original figures of $P$. obes $a$ with the description also based on histological sections.

In these papers, Travassos refers to the measurements of P. obesa according to Diesing (1855) as 3-7 $\mathrm{mm}$ long and 2-5 mm wide. Kloss (1966), mentioned that these measurements must be corrected to 3-7 and 2-5 austriac lines, which correspond to $6.6-15.4 \mathrm{~mm}$ long and $4.4-11 \mathrm{~mm}$ wide.

In 1928, Travassos et al. published a large paper about the helminthological fauna of the freshwater fishes of Brazil. In this paper, the authors refer to new hosts for $P$. obesa, with the same data as Travassos (1922a).

In 1941, Travassos and Freitas reported the presence of adult specimens of $P$. obesa in Astyanax bimaculatus $(=C$. bimaculatum $)$. In the present paper, original measurements of these specimens are presented (Table II).

Pseudoprosthenhystera microtesticulata Kloss, 1966 from A. bimaculatus and A.fasciatus, was considered by Travassos et al. (1969) as a synonym of $P$. obesa. The type specimen of $P$. microtesticulata (no. 2.515) from "Museu de Zoologia da Universidade de São Paulo" has been examined by the authors. It represents, a very young trematode, not well diaphanized. Parenchyma cells, present over the entire body, and more condensed in the borders, were erroneously described by Kloss (1966) as vitellaria, the characteristic used by this author to erect the new genus and species.

$P$. obesa presents a large variation in body shape and size. The large range in body size of worms from the same host and from different hosts, from different localities, collected in different periods is demonstrated in Tables I and II.

The variation in body size of $P$. obesa was also observed by Pavanelli et al. (1992) in three specimens from $S$. maxillosus measuring 8.46 to $18.64 \mathrm{~mm}$ long by 5.65 to $9.88 \mathrm{~mm}$ wide.

Large morphological variation in size and shape of body and in position and shape of testes was already demonstrated by Travassos (1944) in other gall bladder parasites of the family Dicrocoeliidae as in Dicrocoelium dendriticum, Eurytrema coelomaticum, Lubens lubens (=E. (Lubens) lubens), Platynosomum illiciens $(=P$. fastosum), Zonorchis microrchis (Travassos 1944, pls: 3-4, 14, 17, 19-26, 27-30, 53-55).

This high variability was also confirmed in other species of Digenea as in Mesocoelium monas by Freitas (1963), Plagiorchis koreanus and P. verpertilionis by Groschaft and Tenora (1974), Fasciolopsis buski by Roy and Tandon (1993), and others.

The tegumental papillae now observed in $P$. obesa by SEM, were also described in other species as in Gorgoderina vitelliloba (see Hoole \& Michel 1981), Echinostoma revolutum (see Smales \& Blankespoor 1984), Gigantocotyle explanatum (see Ahmad et al. 1988), Zygocotyle lunata (see Irwin et al. 1991), Transversotrema licinum (see Abdul-Salam \& Sreelatha 1992), Fasciolopsis buski (see Roy \& Tandon 1993). At higher magnification we observed in body surface, long and slender structures resembling spines, not referred to previously. As these structures were not visible using light microscopy or by SEM in low magnification, we consider that further observation is required in order to confirm it.

\section{ACKNOWLEDGEMENTS}

To the "Superintendência de Meio Ambiente Aquático", "Centro de Pesquisas" and Dr Carla Canzi from "Itaipu Binacional" for the facilities offered to examine the fish from the Paraná River. To Dr Monika Barth from "Departamento de Virologia, Instituto Oswaldo Cruz", for the aid on the SEM micrographs. To Dr José Jurberg from "Departamento de Entomologia, Instituto Oswaldo Cruz", for the photomicrographs. To Dr José Luiz Moreira Leme from "Museu de Zoologia, Universidade de São Paulo" for the loan of the specimen of Pseudoprosthenhystera microtesticulata studied by Kloss (1966). 


\section{REFERENCES}

Abdul-Salam J, Sreelatha BNS 1992. The surface topography and ultrastructure of the tegument of the ectoparasitic digenean Transversotrema licinum. Zool Anz 228: 48-261.

Ahmad M, Nizami WA, Hanna REB 1988. Topographical studies of two digenetic trematodes of buffalo by scanning electron microscopy. Zool Anz 220: 5964.

Caballero CE, Jimenez FG 1969. Presencia de Prosthenhystera obesa (Diesing,1856) Travas-sos, 1920 (Trematoda, Digenea) en peces comestibles de agua dulce do Mexico. Rev Biol Trop 15: 283-287.

Diesing KM 1850. Systema Helminthum 1. 679 pp.

Diesing KM 1855. Neunzehn arten von trematoden. Denks Akad Wissen, Wien Math Naturw KI 10: 5970.

Freitas JFT 1963. Revisão da família Mesocoeliidae Dollfus, 1933 (Trematoda). Mem Inst Oswaldo Cruz 61: 173-311.

Groschaft J, Tenora F 1974. Some remarks on the morphological variability of the species Plagiorchis vespertilionis (Müller,1780) and Plagiorchis koreanus Ogata,1938 (Trematoda, Plagiorchiidae) parazitizing bats. Acta Universit Agric 22: 115-130.

Hoole D, Mitchell JB 1981. Ultraestructural observations on the sensory papillae of juvenile and adult Gorgoderina vitelliloba (Trematoda: Gorgoderidae). Int J Parasitol 11: 411-417.

Irwin SWB, McCloughlin TJJ, Fried B 1991. Scanning and transmission electron microscopical observations on the tegument of excysted metacercariae and adults of Zygocotyle lunata. J Helminthol 65: 270-274.

Kloss GR 1966. Helmintos parasitos de espécies simpátricas de Astyanax (Pisces, Characidae). 1. Pap Avuls Dep Zool 18: 189-219.

Kohn A, Fernandes BMM 1981. The adult form of Himasthla piscicola Stunkard,1960 and other trematodes from Brazilian freshwater fishes. J Helminthol 55: 85-87.

Kohn A, Fernandes BMM 1987. Estudo comparativo dos helmintos parasitos de peixes do rio Mogi Guassu, coletados nas excursões realizadas entre 1927 e 1985. Mem Inst Oswaldo Cruz 82: 483-500.

Paiva MP 1983. Peixes e pescas de águas interiores do Brasil. Ed. Editerra, 158 pp.
Pavanelli GC, Arana S, Alexandrino de Pérez AC, Machado MH, Matushima ER, Tanaka LK, Dias PG, Sato SK 1992. Parasitose por Prosthenhystera obesa (Diesing,1850) (Trematoda-Callodistomidae) em vesícula biliar de "dourado", Salminus maxillosus (Pisces-Salmininae). SIMBRAq. 7 EMBRAPOA 2. Peruibe Anais: 167-172.

Roy B, Tandon V 1993. Morphological and microtopographical strain variations among Fasciolopsis buski originating from different geographical areas. Acta Parasitol 38: 72-77.

Smales LR, Blankspoor HD 1984. Echinostoma revolutum (Froelich, 1892) Looss, 1899 and Istimiophora melis (Schrank, 1788) Luhe, 1909 (Echinostomatinae, Digenea): Scanning electron microscopy of the tegumental surfaces. J Helminthol 58: 187-195.

Thatcher VE 1991. Amazon Fish Parasites. Amazoniana 11: $263-571$.

Travassos L 1922a. Contribuições para o conhecimento da fauna helmintológica brasileira - XIV. Espécies brasileiras da família Gorgoderidae Looss, 1901. Brasil Médico 36: 17-20.

Travassos L 1922b. Contribuições para o conhecimento da fauna helmintológica brasileira - XVII. Gorgoderidae brasileiras. Mem Inst Oswaldo Cruz 15: $220-234$.

Travassos L 1944. Relatório da excursão do Instituto Oswaldo Cruz ao Município de Santa Teresa, no Estado do Espírito Santo, em agôsto e setembro de 1943. Mem Inst Oswaldo Cruz 40: 121-128.

Travassos L, Freitas JFT 1941. Relatório da terceira excursão à zona da Estrada de Ferro Noroeste do Brasil, realizada em fevereiro e março de 1940. II. Pesquisas helmintológicas. Mem Inst Oswaldo Cruz, 35: 610-634.

Travassos L, Kohn A 1965. Lista dos helmintos parasitos de peixes encontrados na Estação Experimental de Biologia e Piscicultura de Emas, Pirassununga, Estado de São Paulo. Pap Avuls Dep Zool 17: 35-52.

Travassos L, Artigas P, Pereira C 1928. Fauna helmintológica dos peixes de água doce do Brasil. Arch Inst Biol S. Paulo 1: 5-68.

Travassos L, Freitas JFT, Kohn A 1969. Trematodeos do Brasil. Mem Inst Oswaldo Cruz 67: 886 pp. 
Redescription of Prosthenhystera obesa - A Kohn et al. 